\title{
On nanograin rotation by dislocation climb in nanocrystalline materials
}

\author{
Jianjun $\mathrm{Li}^{\mathrm{a}, *}$ A.K. Soh ${ }^{\mathrm{b}}$ and Xiaolei $\mathrm{Wu}^{\mathrm{c}}$ \\ ${ }^{a}$ Department of Engineering Mechanics, School of Mechanics, Civil Engineering and Architecture, \\ Northwestern Polytechnical University, Xi'an, Shanxi, People's Republic of China \\ ${ }^{\mathrm{b}}$ School of Engineering, Monash University Sunway Campus, Malaysia \\ ${ }^{\mathrm{c}}$ LNM, Institute of Mechanics, Chinese Academy of Sciences, No. 15, Beisihuanxi Road, Beijing 100190, \\ People's Republic of China
}

Received 20 November 2013; revised 27 December 2013; accepted 27 December 2013

Available online 4 January 2014

\begin{abstract}
A theoretical model has been developed for nanograin rotation that could be achieved through dislocation climb. The results obtained show that the occurrence of nanograin rotation and the coalescence of grains depend on the external stress level, structure of grain boundary and grain size. Moreover, a critical misorientation parameter that represents the crossover between nanograin rotation and shear-coupled migration of grain boundaries has also been found, based on which a coupling mechanism between nanograin rotation and shear-coupled migration was proposed.
\end{abstract}

(C) 2014 Acta Materialia Inc. Published by Elsevier Ltd. All rights reserved.

Keywords: Nanograin rotation; Dislocation climb; Shear-coupled migration of grain boundaries; Nanograin growth; Strength and ductility

Nanocrystalline (NC) materials are usually strong but brittle compared to their coarse-grained counterparts due to the suppression of conventional dislocation slip in nanograins [1]. Many researchers have made great efforts in the past decades to address the strength-ductility paradox, and numerous NC samples possessing extraordinary strain hardening and ductility have been successfully fabricated [2,3]; their outstanding mechanical behaviors have been attributed to a number of grain boundary (GB)-mediated deformation mechanisms, such as stress-driven nanograin growth [2-5].

Of all the GB-mediated deformation mechanisms, nanograin growth behavior has attracted the greatest scientific interest because of its close correlation with the exceptional ductility of many $\mathrm{NC}$ metals/alloys $[2,3]$. However, the specific mechanism by which nanograin growth enhances the mechanical behavior of $\mathrm{NC}$ materials remains unclear. Experimental studies and molecular dynamics (MD) simulations carried out by researchers have shown that there are two main modes of nanograin growth, i.e. shear-coupled migration

\footnotetext{
* Corresponding author. Tel.: +86 13259738949; e-mail addresses:

jianjunli.mech@gmail.com; mejjli@nwpu.edu.cn
}

(SCM) of grain boundaries $[4,6-10]$ and nanograin rotation (NGR) [5,11-13]. The SCM mode has been identified as a generic mode of plastic deformation [14], as well as an effective toughening mechanism in $\mathrm{NC}$ materials [15], and it can enhance the intrinsic ductility of NC materials considerably [16]. It has also been found in some experiments and mesoscale simulations that the dominant mode of operation depends on grain size, and that these two modes are usually coupled [4]. Nevertheless, the specific mechanism that leads to the above phenomenon remains unclear. Therefore, the main aim of this letter is to develop a theoretical model for studying stress-driven NGR to address the above-mentioned issues. Recent studies have shown that, in the SCM process, a high-angle boundary moves in a similar way to a low-angle boundary, where the displacement shift complete (DSC) dislocations [17], disconnections [18] or structural units [19] composing the former could play the role of lattice dislocations forming the latter. Therefore, it is reasonable for us to postulate that, in the process of NGR, the structural units, DSC dislocations and disconnections in high-angle boundaries could act like the dislocations in low-angle boundaries. As a result, we postulate that NGR could be achieved by dislocation climb for both low- and 
high-angle GBs according to the existing experimental and MD observations $[12,13]$. To simplify the calculation, only a symmetrical tilt boundary that consists of one set of discrete dislocations is considered in the present model.

Consider the rotation of two grains, G1 and G2, that are divided by $\mathrm{GB}$ " $\mathrm{AB}$ " in a deformed elastically isotropic NC specimen, which is subjected to a remote tensile stress $\sigma$ (Fig. 1a). The GB "AB" is modeled as a finite wall of dislocations with identical Burgers vector $\mathbf{b}$ for both high- and low-angle boundaries (Fig. 1b and c). Under external shear stress $\tau$, the leading GB dislocation climbs to the triple junction A $[12,20]$ and dissociates into two Shockley partials, i.e. $\mathbf{b}_{1}$ and $\mathbf{b}_{2}$, which glide along GBs "AC" and "AD", respectively. The two gliding partials meet partials of opposite sign generated by other GBs adjacent to GB "AB" through similar rotating process or extrinsic $\mathrm{GB}$ dislocations, which are the lattice dislocations trapped by the GBs "AC" and "AD", and then annihilate. The GB dislocations remaining in the GB "AB" are assumed to climb following the dissociation of the leading GB dislocation, and finally distribute uniformly along the GB "AB" [20]. Therefore, the spacing between any two GB dislocations is increased from $D_{0}$ to $D_{1}$ (Fig. 1c and d) due to the dissociation of the leading GB dislocation. With the

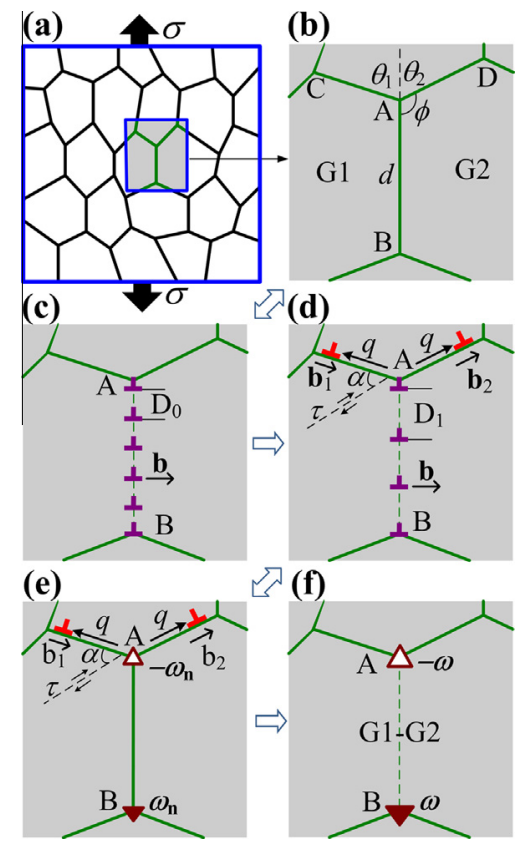

Figure 1. Climb-induced grain rotation process in a nanocrystalline specimen: (a) general two-dimensional microstructure; (b) initial configuration of two grains, i.e. G1 and G2, divided by GB "AB"; (c) equivalent configuration of (b), in which the GB "AB" is modeled as a finite array of discrete dislocations with identical Burgers vector $\mathbf{b}$; (d) under external shear stress $\tau, n$ leading GB dislocations climb one by one to the triple junction $A$ and dissociate into two partials, i.e. $\mathbf{b}_{1}$ and $\mathbf{b}_{2}$, which then glide along the GBs "AC" and "AD", respectively, and annihilate at some points along the said GBs; (e) equivalent configuration of $(\mathrm{d})$, where a disclination dipole (small triangles) is formed due to the loss of GB dislocations; (f) repetition of processes (c) and (d) leads to ultimate coalescence of grains G1 and G2 into one grain, i.e. G1-G2. repetition of the above processes, the original two grains, G1 and G2, would ultimately coalesce into one elongated grain, G1-G2 (Fig. 1f).

In the initial stage, there are two triple junctions between the two grains (G1 and G2) formed by the GB "AB", which is characterized by a tilt misorientation parameter $\theta_{0}$, corresponding to $n_{0}$ GB dislocations. There exist no angle gaps at these two junctions due to the compensation of GB misorientation angles with each other. However, when $n$ leading GB dislocations have climbed, dissociated and disappeared, the misorientation parameter is reduced from $\theta_{0}$ to $\theta_{\mathrm{n}}$, and the resulting angle gaps at the two triple junctions $A$ and $\mathrm{B}$ are $--\omega_{\mathrm{n}}$ and $+\omega_{\mathrm{n}}$, respectively, due to the GB dislocation loss. Therefore, a disclination dipole of arm $d$ and strength $\pm \omega_{\mathrm{n}}$ occurs at GB "AB", as observed in experiments [13]. Once all the GB dislocations have climbed and dissociated, the strength of the disclination dipole is increased up to $\pm \omega$ (Fig. 1f).

Consider the energy change $\Delta W$ produced by the nanograin rotation process in which $n$ GB dislocations climbed to the triple junction A and dissociated (from Fig. $1 \mathrm{~b}$ to e). The rotation process is energetically favorable if $\Delta W<0$. The energy change $\Delta W$ (per unit length along the axis perpendicular to the plane of Fig. 1e) can be expressed as follows:

$\Delta W=W+\Delta E_{d}+E_{\mathrm{int}}^{b_{1} b_{2}}+E_{\mathrm{int}}^{\omega-b_{1} b_{2}}-A_{\mathrm{c}}-A_{\mathrm{g}}$

where $W, \Delta E_{\mathrm{d}}, E_{\mathrm{int}}^{b_{1} b_{2}}$ and $E_{\mathrm{int}}^{\omega-b_{1} b_{2}}$ are the self-energy of the disclination dipole " $\mathrm{AB}$ ", the energy loss due to the dissociation process, the energy resulting from the interaction between the two partials, and the energy that characterizes the interaction between the two partials and the disclination dipole, respectively; $A_{\mathrm{c}}$ and $A_{\mathrm{g}}$ are the work done by the external shear stress $\tau$ for climbing of the GB dislocations along the GB " $\mathrm{AB}$ " and gliding of the partials along the GBs "AC" and "AD", respectively.

The above energy terms for a NC specimen with shear modulus $G$ and Poisson ratio $v$ can be calculated as follows: $W=\mathrm{D} \omega_{n}^{2} d^{2}(\ln (R / d)+1 / 2) / 2[21]$, where $\mathrm{D}=\mathrm{G} /[2 \pi(1-v)], \omega_{n}=\theta_{0}-\theta_{n}=n b / d, b=a / \sqrt{2}, a$ is the lattice parameter and $R$ is the screening parameter for the stress fields induced by the disclination dipole; $\Delta E_{d}=-(3 n-2) \mathrm{D} a^{2}\left(\ln \left(R_{1} / r_{0}\right)+1\right) / 12$, where $r_{0}$ is the dislocation core radius and is assumed to be the same for all the dislocations, i.e. $r_{0} \approx b_{1}$, and $R_{1}$ is the screening length for the long-range stress field of partial dislocation. The energy resulting from the interaction between the two partials can be calculated in the standard way as the work done in generating one partial in the stress field created by another, i.e.

$$
\begin{aligned}
E_{\mathrm{int}}^{b_{1} b_{2}}= & \mathrm{D} b_{1} b_{2}\left[\frac{\cos \Psi}{2} \ln A-\frac{q I}{2 \sin ^{2} \Psi}(2+\cos 2 \Psi) \cos ^{2} \Psi\right. \\
& -\frac{q}{2 A \sin ^{2} \Psi}[x(2+\cos \Psi \cos 3 \Psi) \\
& +q(2+\cos 2 \Psi) \cos \Psi]]_{x=q}^{x=R_{1}}
\end{aligned}
$$

where $b_{1}=b_{2}=a / \sqrt{6}, A=x^{2}+2 x q \cos \Psi+q^{2}, \Psi=\pi$ $-\theta_{1}-\theta_{2}, \quad I=\arctan [(x+q \cos \Psi) /(q \sin \Psi)] /(q \sin \Psi)$. Similarly, 
$E_{\mathrm{int}}^{\left(\omega-b_{1} b_{2}\right.}=\begin{aligned} & -\frac{\mathrm{D} \omega_{n} b_{1} d \sin \theta_{1}}{2} \ln \frac{R_{1}^{2}+2 R_{1} d \cos \theta_{1}+d^{2}}{q^{2}+2 q d \cos \theta_{1}+d^{2}} \\ & -\frac{\mathrm{D} \omega_{n} b_{2} d \sin \theta_{2}}{2} \ln \frac{R_{1}^{2}+2 R_{1} d \cos \theta_{2}+d^{2}}{q^{2}+2 q d \cos \theta_{2}+d^{2}}\end{aligned}$

$A_{c}=\tau d b n \sin (2 \phi-2 \alpha) / 2$

and $A_{g}=-\tau d n\left[b_{1} \cos 2 \alpha+b_{2} \cos (2 \alpha-4 \phi)\right]$. By combining all the above equations, the total energy change $\Delta W$ can be easily determined using Eq. (1) but its expression is not shown here to save space.

A NC Al sample of $G=26.55 \mathrm{GPa}[22], v=0.345$ [22] and $a=0.404 \mathrm{~nm}$ [23] is selected for the following calculations. Other parameters are set as $q=d / 2$, $R=3 d, R_{1}=10 d, \alpha=\pi / 2, \phi=2 \pi / 3$ and $\theta_{1}=\theta_{2}=\pi / 3$.

Figure 2 presents the dependence of energy change $\Delta W$ (in units of $\mathrm{eV} \mathrm{nm}^{-1}$ ) on the normalized number of dissociated GB dislocations $n / n_{0}$; the effects of external shear stress $(\tau)$, initial misorientation parameter $\left(\theta_{0}\right)$ and grain size $(d)$ are shown in Figure $2 \mathrm{a}, \mathrm{b}$ and $\mathrm{c}$, respectively. It can be seen from Figure $2 \mathrm{a}$ that the nanograin rotation process is energetically favorable for all the given stress levels, and that the larger the external shear stress, the more favorable the process is. The effects of $\theta_{0}$ and grain size $d$ are similar to that of $\tau$, but the trends are reversed, i.e. grains with shorter GBs of lower angles (i.e. smaller $\theta_{0}$ and $d$ ) are easier to rotate and coalesce (Fig. $2 \mathrm{~b}$ and c). The above results agree very well with experimental observations $[4,13]$ and MD simulations [12].

Since NGR is one of the two main modes of nanograin growth, it is useful to calculate the critical stress for NGR $\left(\tau_{\mathrm{c}}^{\mathrm{NGR}}\right)$, which is defined as the minimum external shear stress at which the NGR process is energetically favorable, and compare it with that for the SCM process. The critical condition is $\Delta W\left(q=b_{1}, n=1\right)=0$, where $b_{1}$ is the minimum glide distance of the dissociated partials. The expression for $\tau_{\mathrm{c}}^{\mathrm{NGR}}$ can be easily derived from the above equation. However, it is lengthy, so for brevity it is not presented here. The critical shear stress for SCM process $\left(\tau_{\mathrm{c}}^{\mathrm{SCM}}\right)$ can be directly obtained from our previous paper [14]:

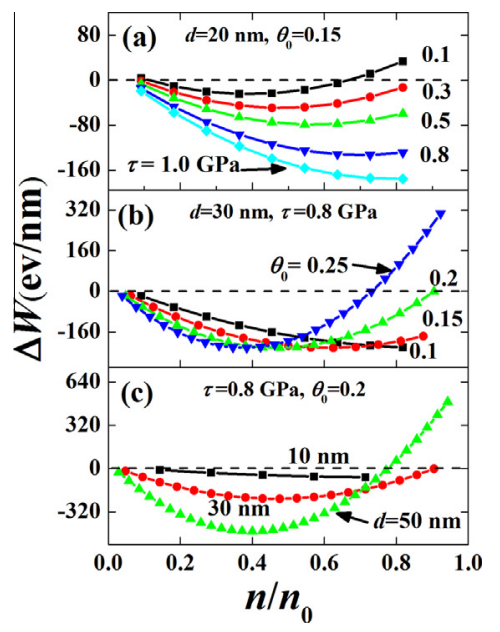

Figure 2. Dependence of energy change $\Delta W$ (in units of $\mathrm{eV} \mathrm{nm}^{-1}$ ) on the normalized number of dissociated GB dislocations $n / n_{0}$ in the case of $\mathrm{NC} \mathrm{Al}$ for various values of external shear stress $\tau$ (a), initial misorientation parameter $\theta_{0}$ (b) and grain size $d$ (c).
$\tau_{\mathrm{c}}^{\mathrm{SCM}}(\beta, d)=\mathrm{D} \omega \frac{2 p^{2} \ln \frac{R}{p}-L_{1}^{2} \ln \frac{R}{L_{1}}-L_{2}^{2} \ln \frac{R}{L_{2}}+2 d^{2} \ln \frac{R}{d}}{d b(1+\beta / \omega)}$

where $\beta$ is the coupling factor that correlates the tangential translation $s$ and the normal migration distance $m$, i.e. $\beta=s / m$, for the SCM process [24], $p=m / \cos \varphi$, $L_{1}=\sqrt{m^{2}+(\beta m+d)^{2}}$ and $L_{2}=\sqrt{d^{2}+p^{2}-2 \beta d m}$, $\varphi=\arctan \beta$ and $m=b$ is the minimum migration distance. Note that the factor 2 in the denominator of the original expression (i.e. Eq. (2) in Ref. [14]) is removed in Eq. (4) in view of the direction difference of the shear stress for the NGR and SCM processes.

Figure 3 shows the variation of critical shear stresses, i.e. $\tau_{\mathrm{c}}^{\mathrm{NGR}}$ and $\tau_{\mathrm{c}}^{\mathrm{SCM}}$ for the NGR and SCM processes, respectively, with respect to grain size $d$ (Fig. 3a) and initial misorientation parameter $\theta_{0}$ (Fig. 3b) in a $\mathrm{NC} \mathrm{Al}$ sample. Two cases are studied in considering the SCM process, i.e. $\beta=0$ and 0.5 . The results obtained in Figure 3(a) show that both $\tau_{\mathrm{c}}^{\mathrm{NGR}}$ and $\tau_{\mathrm{c}}^{\mathrm{SCM}}$ are increased with increasing grain size. Moreover, the coupled shear effect (i.e. $\beta>0$ ) could give rise to a case where the value of $\tau_{\mathrm{c}}^{\mathrm{SCM}}$ is larger than that of $\tau_{\mathrm{c}}^{\mathrm{NGR}}$ first and then becomes smaller. $\tau_{\mathrm{c}}^{\mathrm{NGR}}$ is not expected to remain constant with increasing $\theta_{0}$, whereas $\tau_{\mathrm{c}}^{\mathrm{SCM}}$ does increase with increasing $\theta_{0}$, which results in a transition from the SCM to the NGR process at some critical misorientation parameter, i.e. $\theta_{0 \mathrm{t}}$, as shown in Figure $3(\mathrm{~b})$. This finding indicates that NGR dominates in grains divided by high-angle boundaries while SCM is preferred in those grains associated with low-angle boundaries. In addition, $\theta_{0 t}$ increases as $\beta$ increases from 0 to 0.5 .

Figure 4 plots the variation of $\theta_{0 t}$ with coupling factor $\beta$ for different grain sizes in a $\mathrm{NC} \mathrm{Al} \mathrm{sample,} \mathrm{i.e.}$ $d=10,30$ and $100 \mathrm{~nm}$. The results obtained show that $\theta_{0 \mathrm{t}}$ depends strongly on $\beta$. It increases with increasing $\beta$ up to a certain value, beyond which it decreases. Furthermore, the grain size also has a significant effect on the value of $\theta_{0 t}$, i.e. the larger the grain size, the smaller
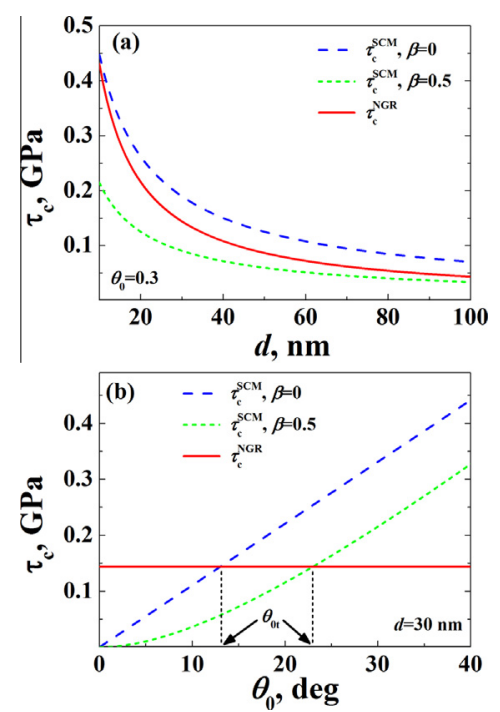

Figure 3. Variation of critical shear stresses $\tau_{\mathrm{c}}$ for the NGR $\left(\tau_{\mathrm{c}}^{\mathrm{NGR}}\right)$ and $\mathrm{SCM}\left(\tau_{\mathrm{c}}^{\mathrm{SCM}}\right)$ processes in a NC Al sample with respect to grain size $d$ (a) and misorientation parameter $\theta_{0}$ (b). 


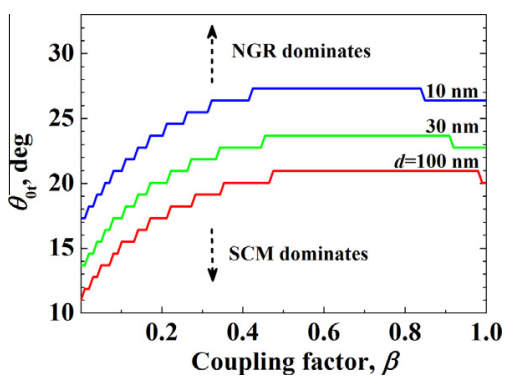

Figure 4. Coupling factor dependent critical misorientation parameter $\theta_{0 \mathrm{t}}$ representing the transition from the NGR to the SCM process for $\mathrm{NC} \mathrm{Al} \mathrm{samples} \mathrm{with} \mathrm{grain} \mathrm{sizes} \mathrm{of} 10,30$ and $100 \mathrm{~nm}$.

the value of $\theta_{0 \mathrm{t}}$. These results suggest that NGR dominates in nanograins $(d<100 \mathrm{~nm})$ since nanocrystalline materials usually consist of high-angle boundaries $\left(\theta_{0}>15^{\circ}\right)[1]$. Thus, it is possible to postulate the following coupling behavior between NGR and SCM: under an external load, NGR occurs first, which leads to a decrease in $\theta_{0}$; subsequently, SCM appears. The cooperative process ultimately results in the nanograin growth behavior in nanocrystalline materials. The above-mentioned coupling behavior is in good agreement with the observations of Sansoz and Dupont [25], who investigated the grain growth behavior of an Al thin film of $7 \mathrm{~nm}$ grain size by indentation at absolute zero $(0 \mathrm{~K})$ temperature using the quasicontinuum method, which resulted in a $\theta_{0 \mathrm{t}}$ of $13^{\circ}$ (see Fig. 2(b-e) of their article). Meanwhile, our model predicts a $\theta_{0 \mathrm{t}}$ of $18^{\circ}$ for the above-mentioned Al sample with a coupling factor of $\beta=0$. These findings can also be used to explain the numerous experimental observations that NGR and SCM are usually intricately interplayed during deformation [4].

In summary, a theoretical model for nanograin rotation is proposed. The results obtained for a $\mathrm{NC} \mathrm{Al} \mathrm{sam-}$ ple show that the NGR process is energetically favorable under a low external shear stress, and can be viewed as a general mode of nanograin growth, as observed in experiments and MD simulations. The analysis of the critical shear stresses for the two modes of nanograin growth, i.e. the NGR and SCM processes, shows that there exists a critical crossover misorientation parameter between the two modes. This finding suggests a possible coupling mechanism of NGR and SCM, which agrees very well with the observations made in molecular static simulations conducted by other researchers. Since both the misorientation parameter $\theta_{0}$ and the coupling factor $\beta$ depend on the specific structure of GBs [24], it is possible to predict which mode (NGR or SCM) would dominate in a NC specimen, and this can be used to optimize its mechanical properties through tailoring of GB structures.
J.L. acknowledges the Start-up Funding for the Newly Recruited High-level Talents from Northwestern Polytechnical University, PR China. A.K.S. acknowledges the support of MOSTI's eScience Fund, Malaysia (Project No. 06-02-10-SF0195) and the Advanced Engineering Programme of Monash University Sunway Campus, Malaysia.

[1] M.A. Meyers, A. Mishra, D.J. Benson, Progress in Materials Science 51 (2006) 427-556.

[2] D.S. Gianola, S. Van Petegem, M. Legros, S. Brandstetter, H. Van Swygenhoven, K.J. Hemker, Acta Materialia 54 (2006) 2253-2263.

[3] X. Wu, Y. Zhu, Y. Wei, Q. Wei, Physical Review Letters 103 (2009) 205504.

[4] M. Jin, A.M. Minor, E.A. Stach, J.W. Morris, Acta Materialia 52 (2004) 5381-5387.

[5] Z.W. Shan, E.A. Stach, J.M.K. Wiezorek, J.A. Knapp, D.M. Follstaedt, S.X. Mao, Science 305 (2004) 654-657.

[6] M.Y. Gutkin, I.A. Ovid'ko, Applied Physics Letters 87 (2005).

[7] I.A. Ovid'ko, A.G. Sheinerman, E.C. Alfantis, Acta Materialia 56 (2008) 2718-2727.

[8] W.A. Soer, J.T.M. De Hosson, A.M. Minor, J.W. Morris, E.A. Stach, Acta Materialia 52 (2004) 5783-5790.

[9] J.T. De Hosson, W.A. Soer, A.M. Minor, Z. Shan, E.A. Stach, S.S. Asif, O.L. Warren, Journal of Materials Science 41 (2006) 7704-7719.

[10] W. Soer, J.T.M. De Hosson, A. Minor, Z. Shan, S. Syed Asif, O. Warren, Applied Physics Letters 90 (2007) 181923-181924.

[11] T. Gorkaya, K.D. Molodov, D.A. Molodov, G. Gottstein, Acta Materialia 59 (2011) 5674-5680.

[12] A.J. Haslam, S.R. Phillpot, H. Wolf, D. Moldovan, H. Gleiter, Materials Science and Engineering A 318 (2001) 293-312.

[13] P. Liu, S.C. Mao, L.H. Wang, X.D. Han, Z. Zhang, Scripta Materialia 64 (2011) 343-346.

[14] J. Li, A.K. Soh, Applied Physics Letters 101 (2012) 241915.

[15] J. Li, A.K. Soh, Scripta Materialia 69 (2013) 283-286.

[16] J. Li, A.K. Soh, Acta Materialia 61 (2013) 5449-5457.

[17] D. Caillard, F. Mompiou, M. Legros, Acta Materialia 57 (2009) 2390-2402.

[18] H. Khater, A. Serra, R. Pond, J. Hirth, Acta Materialia 60 (2012) 2007-2020.

[19] Z.T. Trautt, A. Adland, A. Karma, Y. Mishin, Acta Materialia 60 (2012) 6528-6546.

[20] J. Li, Journal of Applied Physics 33 (1962) 2958-2965.

[21] F. Kroupa, L. Lejcek, Physica Status Solidi B 51 (1972) K121-K124.

[22] W.F. Gale, T.C. Totemeier, Smithells Metals Reference Book, eighth ed., Butterworth-Heinemann, Oxford, 2003.

[23] Y.T. Zhu, X.Z. Liao, S.G. Srinivasan, Y.H. Zhao, M.I. Baskes, F. Zhou, E.J. Lavernia, Applied Physics Letters 85 (2004) 5049-5051.

[24] J.W. Cahn, J.E. Taylor, Acta Materialia 52 (2004) 4887-4898.

[25] F. Sansoz, V. Dupont, Applied Physics Letters 89 (2006) 111901. 